\title{
Implementation and use of the Mental Health Gap Action Programme Intervention Guide (mhGAP-IG): A review of the grey literature
}

\author{
Jessica Spagnolo ${ }^{1,2}$, Shalini Lal ${ }^{3,4,5}$ \\ ${ }^{1}$ Département des sciences de la santé \\ communautaire, Université de Sherbrooke, \\ Québec, Canada \\ ${ }^{2}$ Centre de recherche Charles-Le Moyne- \\ Saguenay-Lac-St-Jean sur les innovations \\ en santé, Campus Longueuil - Université de \\ Sherbrooke, Longueuil, Québec, Canada \\ ${ }^{3}$ École de réadaptation, Faculté de \\ médecine, Université de Montréal, Québec, \\ Canada \\ ${ }^{4}$ Centre de recherche du Centre hospitalier \\ de l'Université de Montréal, Québec, \\ Canada \\ ${ }^{5}$ Douglas Mental Health University Institute, \\ Montreal, Québec, Canada
}

\section{Correspondence to:}

Jessica Spagnolo

Centre de recherche Charles-Le Moyne -

Saguenay-Lac-Saint-Jean sur les innovations en santé (CR-CSIS)

Campus de Longueuil - Université de

Sherbrooke

150 Place Charles Lemoyne

Longueuil

Québec

Canada, J4K 0A8

jessica.spagnolo@usherbrooke.ca
Background The World Health Organization (WHO)'s Mental Health Gap Action Programme (mhGAP)-Intervention Guide (IG) aims to integrate mental health into primary care/community-based settings by equipping non-specialists with tools, training, and support to deliver evidence-based interventions. With the growing popularity of the mhGAP-IG, a systematic review was conducted by Keynejad and colleagues (2018) to identify articles reporting on evidence generated from the implementation and evaluation of the mhGAP-IG in low- and middle-income countries (LMICs). Their review identified peer-reviewed articles and one thesis. In this current review, we report on the implementation and use of mhGAP-IG documented in the grey literature, an important and accessible channel to share information for LMICs.

Methods We searched grey literature databases for documents that reported on the implementation and/or use of the mhGAP-IG or its training modules: ProQuest Dissertations \& Theses Global, the Mental Health Innovation Network (MHIN) database, the WHO website, the mhGAP Newsletter, and the first 10 pages of Google search results. Authors developed and adapted search strategies according to database characteristics. Database searches were completed by November 12, 2019.

Results One hundred and fifty-one $(n=151)$ documents were included in our review. We report on where the mhGAP-IG has been implemented and/or used worldwide. Many types of personnel were trained in the mhGAP-IG and/ or used it in clinical practice. Contextual barriers and facilitators may influence the implementation and/or use of the mhGAP-IG, and we organized these according to structural, organizational, provider, patient, and innovation characteristics. Some information on evaluating the mhGAP-IG was documented in the grey literature. Outcomes included: feasibility of implementing and/or using the mhGAP-IG, its coverage, its impact on the capacities of personnel, patient outcomes, and policies, as well as program costs.

Conclusions This review of the grey literature provides rich experiential knowledge that can complement information documented in the peer-reviewed literature. It is important for researchers conducting reviews on global health/global mental health topics to consider incorporating grey literature search strategies in their reviews. This may not only help to acknowledge the research/dissemination realities of many LMICs, but also to generate findings that reinforce and/or expand those documented in peer-reviewed articles. 
The World Health Organization (WHO) launched the Mental Health Gap Action Programme (mhGAP) in 2008, which aims to further build and scale-up mental health services in LMICs [1]. Specifically, this programme represents the WHO's continued commitment to delivering mental health services through integrated intervention packages and addressing pre-existing barriers to mental health care to facilitate package scale-up [1]. For example, the mhGAP provides a framework for scaling-up mental health interventions by providing guidance on political commitment to mental health care, the assessment of needs and resources, the development of a policy and legislative infrastructure, the "how to" of delivering the intervention package, the strengthening of human resources, the mobilization of financial resources, and monitoring and evaluation [1]. This programme is accompanied by guidelines like the mhGAP-Intervention Guide (IG), which aims to further integrate mental health into primary care and community-based settings by equipping non-specialists with tools, training, and support to deliver evidence-based interventions for what the WHO deems priority mental, neurological, and substance use disorders in low- and middle-income countries (LMICs). These include depression, psychoses, epilepsy, child and adolescent mental and behavioural disorders, dementia, problems related to substance use, and self-harm/suicide [2,3]. The mhGAP-IG was first released in 2010 [2] and is currently in its second version [3].

With the growing popularity of the mhGAP-IG, Keynejad and colleagues (2018) [4] conducted a systematic review to identify articles reporting on evidence generated from its implementation and evaluation in LMICs. Their search yielded 32 peer-reviewed articles and one thesis implemented in 20 countries and/or territories [4]. However, we wondered about the available information on the implementation and use of the mhGAP-IG beyond the peer-reviewed literature (ie, the grey literature). Grey literature is defined as "a range of published and unpublished material which is not normally identifiable through conventional methods of bibliographic control" [5]. Such a realm may be an important and accessible channel for LMICs to share information. For example, studies show inequities in research funding allocation. Limited research funding is usually available for countries with most of the global health problems [6]. Studies also show challenges related to the inclusion of authors affiliated with or from LMICs in peer-reviewed publications [7-10]. Hence, publishing in peer-reviewed journals may be influenced by Global North funding and collaborations. As such, it may be more feasible to disseminate evidence on implementing and using the mhGAP-IG through grey literature sources, such as via blog posts, newsletters, and/or internal reports. Moreover, given the novelty of the mhGAP-IG [2] and its updates [3], as well as the 2017 transfer of its manualized content to an electronic version [11], information on its implementation and use may not yet be published in academic or traditional bibliographic realms.

To our knowledge, the grey literature is a realm previously unexplored for information on the mhGAP-IG. With this paper, our goal was therefore descriptive: to search beyond the peer-reviewed literature to explore and report information about the implementation and use of the mhGAP-IG.

\section{METHODS}

We conducted a review of the grey literature. Aligned with previous studies and methodologies used for conducting a systematic review of the grey literature [12], we first developed a detailed plan for conducting the review, documented in the form of a review protocol. This protocol included the rationale for conducting the review, the search terms, the websites, the limits to the search, the inclusion and exclusion criteria, and a plan for data extraction.

\section{Information sources and search strategies}

We searched the following grey literature databases: ProQuest Dissertations \& Theses Global, the Mental Health Innovation Network (MHIN) database, the WHO website, the mhGAP Newsletter, and the first 10 pages of Google search results. The search strategies were developed by Author 1 and Author 2 and they were adapted according to the characteristics of the databases. Databases were searched starting on 17 September 2019 and they were completed on 12 November 2019.

\section{Proquest Dissertations \& Theses Global}

ProQuest Dissertations \& Theses Global was included as part of our information sources because of recent increases in the support allocated to global mental health training [13]. This database regroups students' final work, which may include any research done on the mhGAP-IG's implementation and use in LMICs. ProQuest Dissertations \& Theses Global was searched using the keywords "Mental Health Gap Action Program*" and its abbreviation "mhGAP," which also serves to target documentation on the IG. The keywords were entered 
within the Search Fields section of the interface. Both keywords were combined with OR to generate the maximum results. We decided to include only these two keywords to keep the search focused on content directly related to the mhGAP.

\section{Mental Health Innovation Network}

The MHIN [14] regroups activities, programs, and/or initiatives in global mental health that aim to improve the lives of those living with mental, neurological, and substance use disorders [14]. The MHIN's team is based at the Centre for Global Mental Health at the London School of Hygiene and Tropical Medicine (UK), and WHO's Department for Mental Health and Substance Abuse (Geneva, Switzerland) [14]. In 2017, the MHIN had more than 3800 members, 232 organizations, and a plethora of resources and contributions (ie, blog posts, manuals, webinars, research, and podcasts) [15]. These resources and documentation may therefore not be reflected in peer-reviewed literature. The MHIN was searched using three keywords, entered one at a time in the Search section of the interface. Keywords include: "Mental Health Gap Action Programme," "mhGAP," and "Mental Health Gap Action Program."

\section{WHO website}

The WHO website [16] was searched because it groups information specifically on the mhGAP and its accompanying intervention tools, such as the mhGAP-IG. A component of the WHO website is the mhGAP Newsletter [17], a newsletter produced to describe ongoing mhGAP activities. When mhGAP activities are being implemented in LMICs, the Department of Mental Health and Substance Abuse at the WHO Headquarters in Geneva aims to cover experiences with such interventions, and the impact they may have on target beneficiaries. The newsletter stories are disseminated globally by email to subscribers, as well as on the WHO website [17]. The WHO website was searched in two ways. First, the mhGAP newsletters section was retrieved by typing "mhGAP Newsletters" in the search section of the WHO website. At the time of the search (between 17 September 2019 and 12 November 2019), 19 editions of the mhGAP newsletter were listed on the WHO website, ranging from January 2011 to April 2019. Each of these newsletters were searched manually. The WHO website was also searched using the keyword "Mental Health Gap Action Program" in the search section of the website.

\section{Google}

We searched the first 10 pages of Google search results for relevant documents and/or information on the implementation and use of the mhGAP-IG. Google is a common search engine for grey literature reviews, and we searched the database using three keywords, as follows: "Mental Health Gap Action Programme" OR "mhGAP" OR "Mental Health Gap Action Program." Keywords were combined with OR to generate the maximum results.

\section{Document selection, data extraction, and analysis}

Data selection, data extraction, and analysis were primarily conducted by Author 1, and both process and outcomes were reviewed and discussed at several stages with Author 2. First, all documentation generated by the search strategy was included in a data extraction file using Excel software, each tab regrouping the results for each of the databases included. The first author and a research assistant conducted the search strategy. They then removed the duplicates in each of the tabs. Second, the first author applied the inclusion criteria to each of the items generated by the search strategy. Inclusion criteria were documentation that described the implementation and/or use of the mhGAP-IG. We also included: documentation that mentioned the mhGAP with for example the words training, guidelines, or that related to capacity-building and documentation that referred to modules of the mhGAP-IG; as well as documentation that highlighted prospective use. We excluded peer-reviewed literature that we found through our grey literature search. If results met these eligibility criteria, they were included in the paper. There were six documents that the first author was unsure about (ie, labelled as 'maybe'; these were then reviewed in detail by the second author, as part of the decision-making process). This resulted in 151 documents included in the review. Third, for these included documents, the first author extracted and aggregated information related to the following themes: country of implementation; type of personnel who used the mhGAP-IG and in what setting; mhGAP-IG modules used; information on mhGAP-IG adaptation; contextual factors that may influence the implementation and/or use of the mhGAP-IG; and information on type of outcomes used to assess mhGAP-IG's implementation and impact. These categories were used to organize the results section of the paper. 


\section{RESULTS}

The search and selection process are described in Figure 1. One hundred and fifty-one $(n=151)$ documents were retained. A full list of included documents may be found in the Table S1 in the Online Supplementary Document.

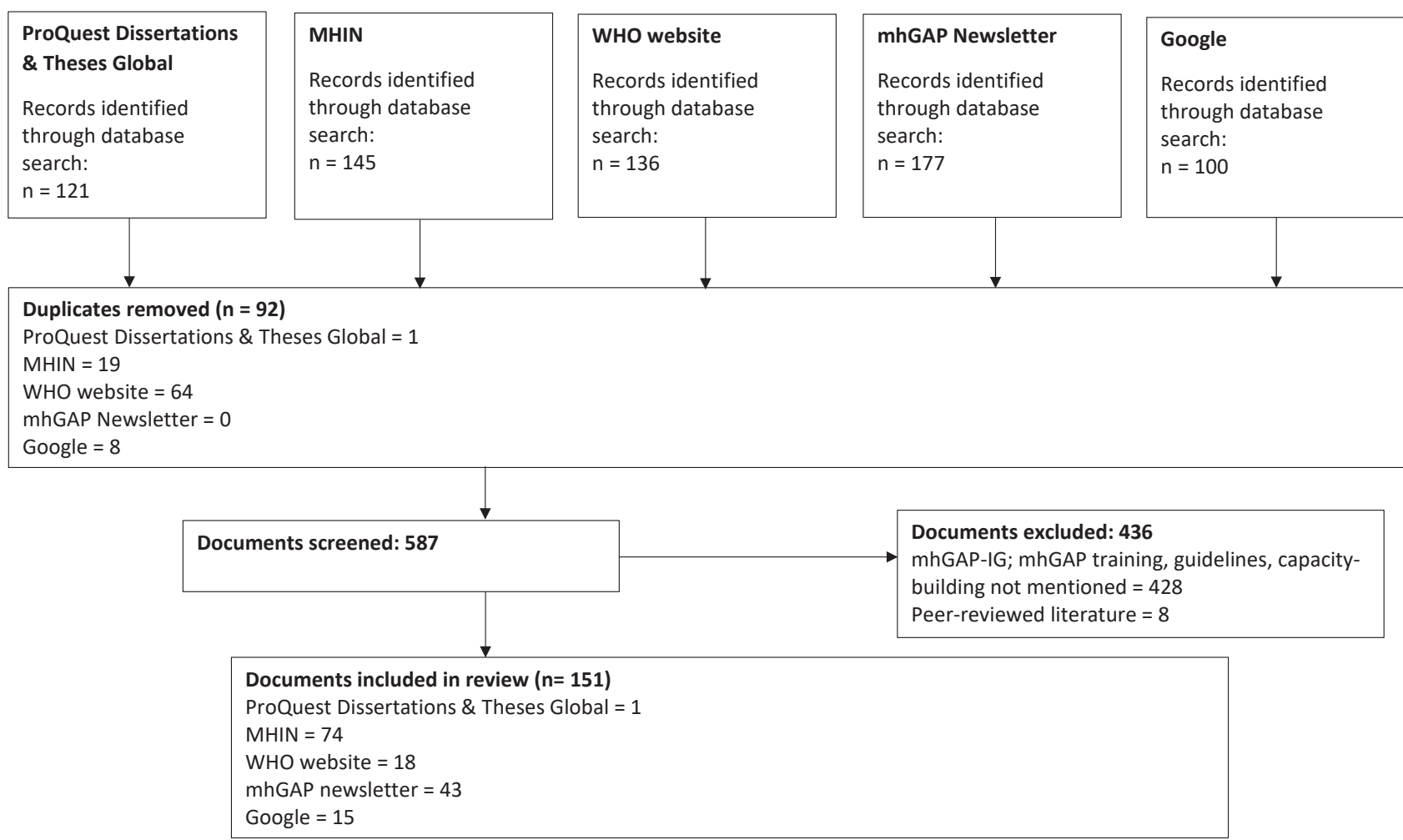

Figure 1. Flowchart identifying documents to be included in the review (Adapted PRISMA Flow Diagram). MHIN - Mental Health Innovation Network, WHO - World Health Organization, mhGAP - Mental Health Gap Action Programme, mhGAP-IG - Mental Health Gap Action Programme Intervention Guide.

\section{Country of implementation}

The documentation we reviewed pertained to the implementation and/or use (including prospective use) of the mhGAP-IG in 92 countries/territories, primarily countries classified as LMICs according to the World Bank's Income Categories [18]. Specifically, our findings show that $18.68 \%$ of countries implementing and/or using the mhGAP-IG are classified as low-income, $31.87 \%$ as lower-middle-income, $31.87 \%$ as upper-middle-income, and $17.58 \%$ as high-income. To generate these percentages, we relied on a denominator of 91 countries/ territories, as one country/territory included in our review was not listed in the World Bank Income Categories [18]. In addition, our findings show that most countries implementing and/or using the mhGAP-IG according to the grey literature were those of the WHO Region of the Americas (33.33\%). Other countries were represented as follows: 28.74\% from the WHO African Region; 11.49\% from the WHO Eastern Mediterranean Region; 11.49\% from the WHO European Region; 8.05\% from the WHO South-East Asia Region; and 6.90\% from the WHO Western Pacific Region. To generate these percentages, we relied on a denominator of 87 countries/territories, as five countries/territories included in our review were not identified on the website for their respective WHO Region [19]. A full list of countries identified by our review is included in the Table S2 in the Online Supplementary Document.

Of note, in one mhGAP Newsletter (June 2014), there was mention of six Caribbean countries and 14 countries of the WHO Region of the Americas, yet the specific countries were not explicitly listed. Similarly, one retrieved document from the WHO website and one from Google mentioned 14 Caribbean and 13 Latin American countries [20], yet these were not explicitly listed. These countries may have been omitted from the country list (Table S2 in the Online Supplementary Document). The same applies to the five Francophone and Anglophone countries listed but not explicitly mentioned in one Google document [20]. 


\section{Types of users and settings}

The mhGAP-IG was used as a training tool and/or in clinical practice to help personnel detect, treat, and manage mental, neurological, and substance use disorders, as well as refer people presenting with these conditions to specialists. The following types of personnel were engaged with the mhGAP-IG, either in training and/or by using it in clinical practice: 1) national entities like officials from the Ministry of Health, national network managers, and staff from the District of Health; 2) non-specialists like nurses, primary care physicians, physician assistants, resident physicians (for example, emergency medicine trainees), social workers, paramedics, public safety officers, community workers, auxiliary staff, community health extension workers, midwives, aid workers, and human immunodeficiency virus (HIV) lay counselors; 3) specialists like psychologists, psychiatrists (some of which then became trainers), mental health nurses, mobile mental health teams, community mental health workers, psychosocial workers, and pediatricians; 4) health planners and administrators including directors of nursing, community welfare officers, and chief welfare officers; and 5) community champions/leaders, like health and nutrition committee members, traditional birth attendants, community resource persons, volunteers, mothers, family members of children affected by mental health problems, teachers, religious leaders, and traditional healers. There was also mention of the mhGAP-IG training of master trainers to further the implementation process.

In terms of settings, the mhGAP-IG was delivered in primary care and community-based settings like primary care clinics, family medicine centers, as well as in the prison system, hospitals (district, provincial, and teaching, as well as outpatient facilities), and emergency/conflict settings like refugee camp health facilities. The mhGAP-IG was also offered in formal education settings: for example, by integrating its modules into medical undergraduate curricula at universities (Ethiopia, Somalia). In another example, medical students from the United Kingdom were paired with medical students from India and Somaliland to e-learn the mhGAP-IG modules, promoting peer-topeer learning across cultures [21,22]. In addition, the mhGAP-IG was incorporated into the teaching curricula to train students in Nepal by didactic video lecture [23] and to train community mental health workers in Haiti through a program called Psy-pour-Haiti [24]. The mhGAP-IG was also included in leadership programs as part of continuing education. For example, in Libya, primary mental health care officers were offered a newly minted six-month diploma programme that included modules of the mhGAP-IG. The University of Ibadan in Nigeria coordinates the Mental Health Leadership and Advocacy Programme (mhLAP) where mental health leaders, officials, and advocates from The Gambia, Ghana, Liberia, Nigeria, and Sierra Leone can further their mental health learning, including of the mhGAP-IG. Through this programme, for example, national master trainers could be trained to use the mhGAP-IG, after which they would train non-specialists in their respective countries.

\section{mhGAP-IG modules}

mhGAP-IG modules that were used in the documentation that we reviewed include: the general introduction to the mhGAP-IG; general principles of care; epilepsy; developmental and behavioural disorders in childhood and adolescence; conditions specifically related to stress (acute stress, posttraumatic stress); dementia; depression; child mental health; psychosis; schizophrenia; problems related to alcohol and drug use; self-harm/suicide; and other significant, unexplained emotional or medical complaints. Use of the mhGAP implementation tools and the operations manual was mentioned. Beyond the mhGAP-IG (but included in complementary training), some trainees received courses on psychotherapeutic interventions like cognitive behavioural therapy, family therapy, and counseling skills.

\section{mhGAP-IG adaptations}

The mhGAP-IG along with its accompanying training material (ie, PowerPoints, facilitator and participant guides) are standardized documents that the WHO suggests adapting prior to use $[2,3]$. In the documentation that we reviewed, there was mention of mhGAP-IG contextualization to the local context (ie, translation to local languages and/or adaptation to meet realities of primary and community-based health care settings, affected displaced populations and host communities, or humanitarian settings) in 31 countries (Table S2 in the Online Supplementary Document). Some initiatives mentioned that adaptations were made to specific mhGAP-IG training modules and their accompanying training materials so that they fit into specific time frames and/or so that the mhGAP-IG could be used via online platforms.

\section{Contextual factors influencing implementation and use}

The mhGAP-IG was implemented and used within specific contexts. Contextual factors that may have influenced the implementation and use of the mhGAP-IG were highlighted in some of the documentation that we reviewed. We organized examples of these factors according to the following categories: structural (ie, the sociocultural 
context in which an organization is nested), organizational (ie, aspects related to the organization in which an innovation like the mhGAP-IG is implemented), provider (ie, aspects related to the provider that implements the innovation within the organization), patient (ie, aspects related to the people exposed to the innovation, such as health beliefs, personality traits, etc.), and innovation (ie, aspects related to the innovation to be implemented, such as quality, usefulness, etc.). These categories were inspired by Chaudoir and colleagues (2013)'s framework [25]. Table 1 illustrates examples of the barriers and facilitators to mhGAP-IG implementation and/or use.

Table 1. Contextual factors (barriers and facilitators) that may influence mhGAP-IG implementation and use

$\begin{array}{ll}\text { FACTORS } & \text { BARRIERS } \\ & \begin{array}{l}\text { Political } \\ \text { - political turnover (ties with specific political leaders may have encouraged } \\ \text { mhGAP-IG implementation and use) }\end{array} \\ & \begin{array}{l}\text { - challenging working relationships with certain policymakers and govern- } \\ \text { ment staff }\end{array} \\ & \text { - high conflict, political tension, humanitarian crisis (ie, displacement) } \\ \text { - financial crisis } & \\ & \text { - political tension } \\ & \text { - limited funds allocated to mental health } \\ & \text { - no (or outdated) mental health plans to address mental, neurological, and } \\ \text { substance use disorders (if a strategy is available, it may not be adopted and/ } \\ \text { or operationalized) } \\ \text { - centralized systems of care (challenging reach of mental health services) } \\ \text { Struc- } \\ \text { tural rastructure }\end{array}$

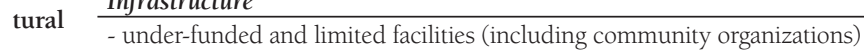
FACILITATORS

Political

- support from and collaborations with the Ministry of Health and/or other national entities

- new mental health legislation (or strategies) to further encourage funding for mental, neurological, and substance use disorders and health system strengthening in primary care and/or community-based settings

- the revision of national mental health treatment protocols

- the creation of a national mental health committee at the level of the Ministry of Health

- the inclusion of mhGAP-IG training modules as part of the national medical training curricula

- national workshops to disseminate pilot findings of mhGAP training, which can encourage scale-up

\section{Infrastructure}

- leveraging available human resources and care settings, from primary to

- fragmented services

specialized care

- poorer quality of health care services

- difficulty accessing health care facilities (eg, persistent conflict)

- limited human resources (including mental health specialists to provide

non-specialists with support)

- lack of a national committee for implementing the mhGAP training

- limited and/or high cost of medications

- inconsistent and/or unavailable Internet coverage (for mhGAP-IG imple-

mentation and use that rely on virtual tools)

Socio-cultural

Socio-cultural

- stigma against mental illness $\quad$ - community mental health awareness

Geographical

- difficult terrain to access clinics

- natural disasters (ie, earthquakes, typhoons) making physical access to care more difficult

\section{Infrastructure Infrastructure}

- understaffing at health facilities where training was implemented

- frequently out-of-stock medication within health care clinics

- planning for sustainable support and supervision mechanisms

- lack of appropriate space and privacy for mental health care delivery

- developing an appropriate referral system to coordinate care

- defining roles and responsibilities for all stakeholders involved in the mhGAP-IG implementation

- questionable fidelity of data collected due to poorer documentation, lim- - training many types of non-specialists within the health care centers ited electronic data collection systems, and time-consuming nature of data reporting and collection

- lack of supervision or support to implement the mhGAP training in health - providing mhGAP-based training in health care centres with available medcare centers ication

- leveraging already used technology to participate in training and to treat patients

Organi-

zational

Collaborations
- insufficient intersectoral collaboration

- employing a cascade model of training

\section{Collaborations}

- receiving support from key organizations (WHO, PAHO), universities, non-governmental organizations, psychiatrists - to facilitate regular supervision and ongoing feedback to trainees)

- difficulties in the referral processes between organizations, including chal-

lenges with continuing of care - from specialized settings to community follow-up

Vision

- low mental health priority in health care centres

Vision

- limited mental health leadership within health care centres, affecting provid-

ers' engagement in mental health care delivery and their use of the mhGAP-IG

- fostering a vision that adopts evidence-based services

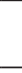


Table 1. Continued

\begin{tabular}{|c|c|c|}
\hline FACTORS & BARRIERS & FACILITATORS \\
\hline \multirow{7}{*}{ Provider } & Non-specialists & Non-specialists \\
\hline & $\begin{array}{l}\text { - heavy workload, with competing priorities in daily practice beyond men- } \\
\text { tal health care }\end{array}$ & $\begin{array}{l}\text { - non-specialists' willingness to include mental health as part of routine clin- } \\
\text { ical tasks }\end{array}$ \\
\hline & - professional attrition (retirement, promotion, transfers) & - a reliance on and continuous use of feedback \\
\hline & - negative bias against some treatment interventions listed in the mhGAP-IG & - intervention 'buy-in' \\
\hline & - staff rotation & $\begin{array}{l}\text { - an emphasis on training newly graduated professionals to further foster } \\
\text { acceptance of mental health care delivery in primary care and/or communi- } \\
\text { ty-based settings }\end{array}$ \\
\hline & Mental health specialists & \\
\hline & - reluctance to recognize non-specialists' role in mental health care & \\
\hline Patient & - experiences of mental health stigma & - satisfaction with services received by trained staff \\
\hline \multirow{3}{*}{$\begin{array}{l}\text { Innova- } \\
\text { tion }\end{array}$} & - unavailability of accompanying training materials in local languages & $\begin{array}{l}\text { - adaptability of the mhGAP-IG and accompanying training tools to local } \\
\text { contexts and needs }\end{array}$ \\
\hline & - omitting to implement the supervision component post-mhGAP training & $\begin{array}{l}\text { - the training's clinical utility (including interactive components like group } \\
\text { discussions and role plays) and user-friendliness }\end{array}$ \\
\hline & - not enough mental health training to meet all mental health learning gaps & - relying on technology to provide training \\
\hline
\end{tabular}

mhGAP-IG - Mental Health Gap Action Programme Intervention Guide, mhGAP - Mental Health Gap Action Programme, WHO - World Health Organization, PAHO - Pan-American Health Organization

Of note, the Pan-American Health Organization (PAHO)'s Virtual Campus for Public Health (VCPH) was described as facilitating training on using the mhGAP-IG. This platform provides opportunities for synchronous and asynchronous learning on many diverse topics related to public health, including the mhGAP-IG [26]. This innovative way of teaching the mhGAP-IG was conducted in Caribbean countries, as well as in Latin American countries like Guyana. With this platform, trainees could learn at their own pace, as well as connect with other participants and instructors. In Mexico, combined periods of distance learning and in-person seminars were also employed. In addition, Colombia used the VCPH to group the expertise of four universities to teach the mhGAP-IG. The WHO Collaborating Centre for Mental Health Services Research and Training from Spain's Universidad Autónoma de Madrid (UAM) Department of Psychiatry also used this online platform to provide mhGAP-based training in Mexico. The VCPH content is available in English, French, Spanish, and Portuguese [26].

Beyond the VCPH, training and/or clinical support to non-specialized mental health care staff was provided using other technological means. Some examples include the use of didactic videos to provide lecture-based training of mhGAP-IG content in Nepal [23]; a mobile technology to train, supervise, support, and monitor application of the mhGAP-IG's depression module in Kenya [27]; an interactive voice response and an avatar-assisted training and supervision system related to developmental delay for families and volunteers in Pakistan (The FaNs for Kids Project, a pilot project for sustainable scale-up [28]); a mobile-based clinical decision support tool based on the mhGAP-IG's algorithm to help staff identity and manage mental disorders, and a mobile follow-up for trainees in India (SMART Mental Health) [29]; and Skype for mhGAP-IG trainee supervision in Nigeria and offered by Global North partners. Other examples included cell phone call reminders for community health workers involved in learning the mhGAP-IG and the provision of video-conferencing and educational CDs to support self-learning training in Afghanistan; tablets provided during the mhGAP-IG training to further interactive learning and their use in clinical practice in the Union of Comoros; the mobile version of the mhGAP-IG child and adolescent depression module to support staff providing care in South Africa and Zambia [30]; and the electronic version of the mhGAP-IG on a mobile device for routine use in community-based settings in Nepal and Nigeria (Emilia project) [31,32].

\section{mhGAP-IG evaluation}

Some documents we reviewed mentioned outcome data. We opted to qualitatively report on examples of the types of outcomes mentioned in the documents that we reviewed, given that outcome data was reported heterogeneously across initiatives. These outcomes are summarized in Table 2.

\section{DISCUSSION}

As shown by our review of the grey literature, we identified over 90 countries in which the mhGAP-IG was implemented and/or used, including for its prospective use. Our review highlights the wide span of the mhGAP-IG's implementation and use and shows a plethora of experiential knowledge available from the grey literature, including news articles about peer-reviewed papers [33]. While the details provided in the documentation found were variable and may be limited in some cases, many of the documents we reviewed 
Table 2. Outcome measures for the mhGAP-IG implementation and use, with associated examples and tools, if mentioned

EVALUATION

OUTCOMES

EXAMPLES

Developing and implementing an mhGAP-based training and/or mental health care plans includ-

ing the mhGAP-IG

Implementing and using the mhGAP-IG (electronic vs paper versions)

- staff acceptability

Feasibility $\quad$ - non-specialists' ability to provide evidence-based treatment according to patient symptoms

- people with depression being identified by non-specialists

- affordability and cost-effectiveness

- level of clinical support and supervision to trainees

- level of mental health stigma among non-specialists

Number of personnel trained to use the mhGAP-IG

Number of people who sought care by trainees or personnel using the mhGAP-IG in clinical practice

Availability of evidence-based services at facilities, including those based on the mhGAP-IG

Treatment gap, measured pre- and post-mhGAP-IG implementation

Treatment coverage using the e-mhGAP-IG and the paper version

Number of personnel per health care facility who learned to use the mhGAP-IG

Number of personnel who received support and supervision sessions by specialists

Availability of psychotropic medication supply

Number of prescriptions including by mhGAP-IG trainees

Healthcare utilization by families

Level of mental health integration in primary care sites including organizational-level integration of International Medical Corps Primary Health Care mental health, to make care more accessible

Mental health knowledge

Mental health attitudes Integration Checklist

mhGAP knowledge questionnaire

MICA; SDS; IAT

Mental health confidence

Mental health self-efficacy

Self-reported mental health practice, changes in practice, enhancement of skills

- screening, accuracy of detection

- diagnosis

Impact on - patient treatment and management, including prescribing and offering psychosocial care

personnel - referrals

Therapist and non-specialist competence

ENACT scale; TASC-R

Satisfaction with training (feedback about the program)

Quality checks

Mental health conditions treated by personnel

Costs (travel costs for people accessing care and treatment costs for families)

Quality of life

Disability

Patients' integration back into the community

Experience of stigma

Patient follow-up (returning to appointments)

Functioning, overall symptoms, well-being

Impact on Depressive symptoms

patient Number of seizures and their level of severity

outcomes Suicidal thoughts

Symptom remission and recovery

Socio-emotional well-being of children

Impact on children's families (stigma and parental distress, utilization of health care services)

Patient satisfaction

Decrease in mortality (eg, by suicide)

Psychotropic medication supply

Annual mental health budget allocation (eg, transition from a previously WHO-supported drug

treatment and care system to one that is locally funded)

Impact on Improvements in human resources

policies Creation of a health information system for depression and other medical conditions

Political interest in mental health (eg, the establishment of a mental health unit at the Ministry of

Health and the launching of national mental health policies)
On-the-job supervision checklists; Institutional quality checklists, including verification that appropriate records are being kept for each patient, and a review of client diagnosis and care plans; Quality Improvement measures

WHO Quality of Life-BREF; European Quality of Life Scale

WHO-DAS; WHO-DAS-CHILD

DISC-12

HSCL; HTQ; GHQ-12

BDI; ZLDSI; PHQ-9

Health of Nations Outcomes Scale; Qualitative interviews: people with lived experiences, family caregivers, health care providers, community leaders

Verona Service Satisfaction Scale; mhGAP training reports 
Table 2. Continued

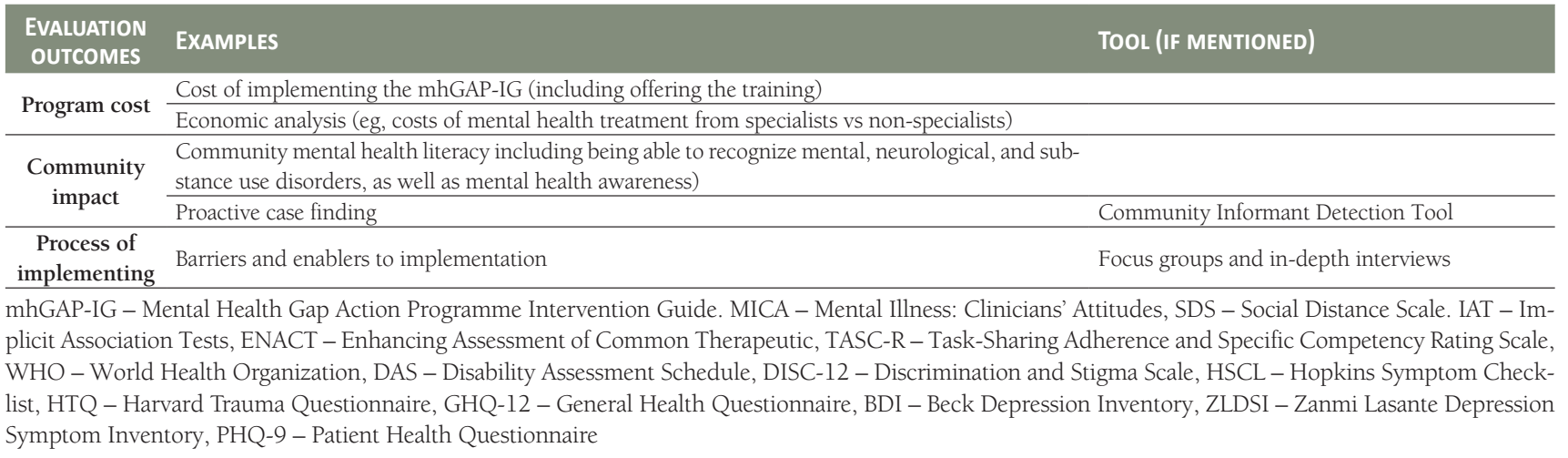

provided valuable information. Our review highlights the types of mhGAP-IG modules and personnel who use the mhGAP-IG. For example, we identified a wide range of modules being used, from modules on common mental disorders (depression, anxiety) to conditions considered more severe and complex (dementia, psychosis, alcohol and substance use disorders, self-harm/suicide). Training in such mental, neurological, and substance use disorder modules considered more severe and complex may be a way to help address mental health stigma [4,34-36], which was identified in the documents and can be a contextual factor that challenges the implementation and use of the mhGAP-IG. We also found that several types of personnel participated in mhGAP-IG training and/or used it in clinical practice, most being non-specialists in mental health care. This range of personnel who may be working in different settings highlights the collaborative approach that may be favoured in the mhGAP-IG to offer care [2,3]. It also emphasizes the importance of task-sharing, the increased involvement of non-specialists in mental health care and with the support and supervision of specialists [37,38]. One stakeholder group identified by our search was traditional healers. Traditional healers may be consulted for mental health care and might therefore be valuable resources for the detection of mental, neurological, and substance use disorders [39,40]. Our review found that many countries have engaged in contextual adaptations of the mhGAP-IG or accompanying training material. These adaptations are encouraged by the WHO and may be a way to foster local ownership of the mhGAP-IG implementation and use to encourage its scalability [4,41-43].

Our review identified contextual factors that may influence the implementation and use of the mhGAP-IG and examples of the types of outcomes that were considered and/or used to assess its impact. This experiential knowledge may be beneficial to countries interested in mhGAP-IG implementation, especially since this documentation in the grey literature may be more accessible in LMICs. For example, it can highlight practical and policy changes and/or encourage the reinforcement of facilitators that may further support implementation and use of mhGAP-IG guidelines, as well as task-sharing in respective countries. In addition, program implementors and/or evaluators can mobilize our findings from the grey literature to help identify types of outcomes which might not necessarily be included in the mhGAP training packages, in order to assess impact [44]. Our findings also reinforce and expand on evidence related to contextual barriers and facilitators from the peer-reviewed literature, some of which are more largely discussed as factors influencing the integration of mental health and/or mental health programmes into primary care and/or community-based settings [45], one of the mhGAP objectives.

Our findings highlight that some mhGAP-IG initiatives relied on technological tools to support training and/ or guideline implementation into clinical practice. In the context of COVID-19, virtual means to provide mental health training and/or deliver mental health services are being encouraged [46]. We can learn to offer virtual mental health training and support to non-specialists on their mental health care delivery from the experiential knowledge gained by mobilizing existing platforms and infrastructure, such as the VCPH and mobile applications like the mhGAP-IG's electronic version. These virtual means may have enormous potential in supporting/offering clinical care and/or in training personnel $[47,48]$, including during and after the COVID-19 pandemic. We do acknowledge the importance of future research on implementing and using technology for delivering mental health care services, including exploring the barriers and facilitators of using technology to implement mhGAP-IG initiatives, as well as such technology's effectiveness in comparison to using non-virtual means, as proposed by the Emilia project [31,32]. 
We experienced several challenges in conducting this review of the grey literature that are noteworthy to mention to generate further discussion on grey literature methodology, especially for the global health literature context. First, many of the documents did not have authors or dates. Neither did they all provide the name of the initiative that mobilized the mhGAP-IG. Thus, it was often difficult to associate authors with our findings to identify duplicates across platforms and to group documentation under an umbrella initiative. We opted to refrain from quantifying beyond an initial reporting on the number of retained documentation. Our review therefore aims to provide a qualitative and descriptive portrait of what we found. However, this grouping across search platforms, even if with only a couple of initiatives, could be an interesting exercise for future grey literature reviews, as it can help to improve understanding of how knowledge on implementation and use of the mhGAP-IG is being disseminated and through which platforms. Second, we noticed that information provided in the grey literature was heterogeneously reported within and across platforms. There was information that we originally planned to report in the findings but opted not to due to this inconsistency (ie, outcomes to comment on program effectiveness, number of non-specialists using the mhGAP-IG, number of training hours/days, number of people consulting mhGAP-based trained personnel, etc.). Lastly, we noticed that the content from some website links was sometimes inaccessible and/ or no longer available, a challenge inherent in the grey literature: content and links may not be available as permanently as they are in peer-reviewed journals.

Limitations to conducting our review of the grey literature should also be noted. First, many of the initiatives came with references and additional resources. However, given limits to our research team, we opted not to review these references. These may have helped identify additional countries where the mhGAP-IG was implemented, and/or may have provided additional information on the mhGAP-IG's use. Second, one of the hits identified on Google was the mhGAP hashtag on Twitter (\#mhGAP). We did not search social media platforms such as Twitter and Facebook; however, searching these platforms may be promising avenues for future grey literature searches, given the increased use of social media for health related purposes in LMICs [49] and the ability to share information on mhGAP-IG implementation and use quickly and informally. Third, data extraction was conducted primarily by the first author, and this may have been subject to human error. Moreover, results on barriers and facilitators to the implementation and use of the mhGAP-IG were not always reported as such in the initiatives. Some barriers and facilitators were discussed as country/territory contextual factors, and the first author regrouped these as potential factors that may influence the implementation and use of the MhGAP-IG, a similar exercise explored elsewhere [50]. The identification of barriers and facilitators, as well as their grouping according to categories included in Chaudoir et al. (2013)'s [25] framework, may be subject to interpretation. The same should also be mentioned for the grouping of the outcome measures; these were done at the discretion of the first author. Nonetheless, all grouping of categories and synthesis of findings was reviewed by the second author and discussed at team meetings to help reduce bias and enhance clarity of the summaries. Fourth, it is worthy to note that some documents reported the use of the mhGAP-IG in parallel to other training material and/or initiatives, in the form of a package. For example, the PRIME project [51-54] relied on different-level initiatives, including the mhGAP-IG as a faculty-level support for providers to treat mental, neurological, and substance use disorders. Specifically, the mhGAP-IG was used as a part of mental health care plan packages. The specific use of the mhGAP-IG was sometimes difficult to tease out among these packages. Lastly, many of the grey literature sources we used to search for relevant materials were websites such as the MHIN website and the WHO website. Websites are subject to change in terms of features (eg, updates to their search engine), which is an important factor to consider for future replication or update of this review.

\section{CONCLUSIONS}

Our overview of the grey literature provides rich experiential knowledge. Searching the grey literature may not only acknowledge the research and dissemination realities of many LMICs, but it can generate results that reinforce and/or expand peer-reviewed findings and discussion. We therefore encourage researchers conducting reviews on global health and global mental health topics to consider incorporating grey literature search strategies in their reviews. 


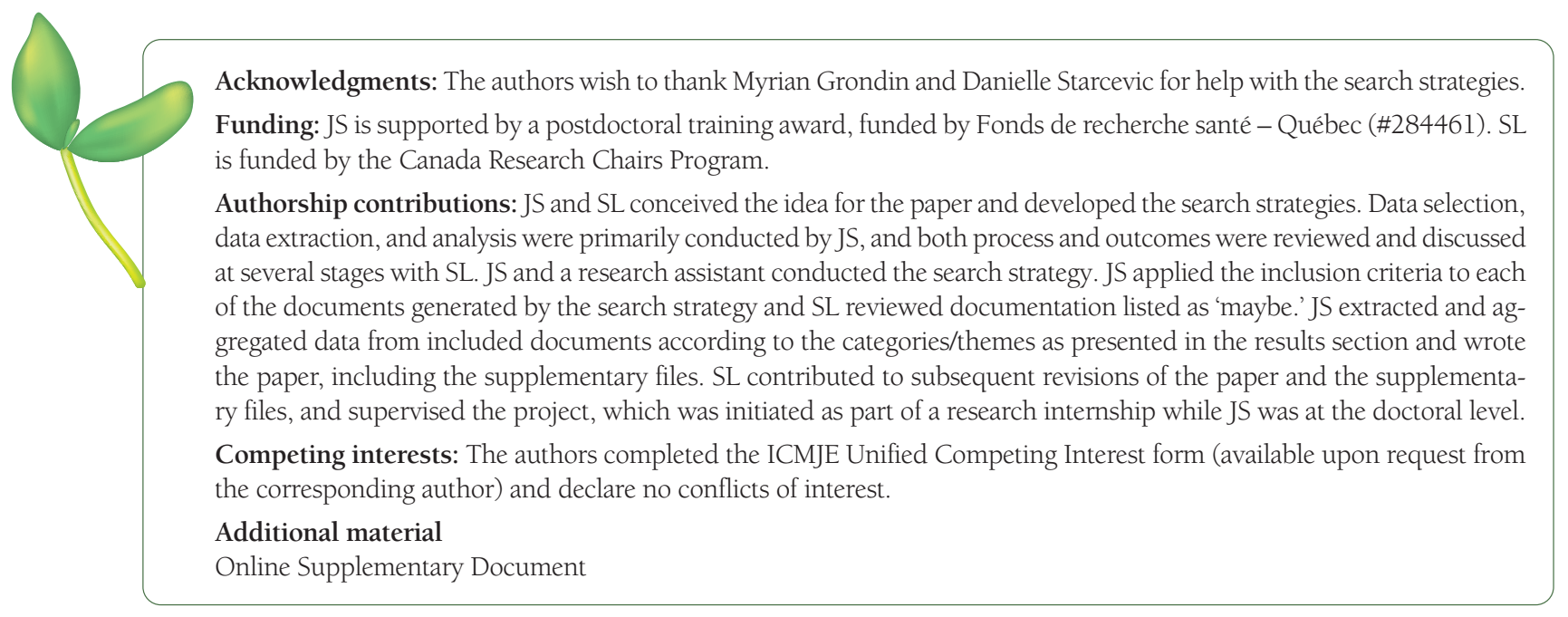

1 World Health Organization. mhGAP Mental Health Gap Action Programme: scaling up care for mental, neurological, and substance use disorders. Available: https://www.who.int/mental_health/evidence/mhGAP/en/. Accessed: 15 November 2020.

2 World Health Organization. mhGAP Intervention Guide for mental, neurological and substance use disorders in non-specialized health settings, version 1.0. Available: https://www.who.int/mental_health/publications/mhGAP_intervention_guide/en/. Accessed: 15 November 2020.

3 World Health Organization. mhGAP Intervention Guide for mental, neurological and substance use disorders in non-specialized health settings, version 2.0. Available: https://www.who.int/publications/i/tem/mhgap-intervention-guide---version-2.0. Accessed: 15 November 2020.

4 Keynejad RC, Dua T, Barbui C, Thornicroft G. WHO Mental Health Gap Action Programme (mhGAP) Intervention Guide: a systematic review of evidence from low and middle-income countries. Evid Based Ment Health. 2018;21:30-4. Medline:28903977 doi:10.1136/eb-2017-102750

5 Hart C. Doing a Literature Search: A comprehensive guide for the social sciences. London: Sage Publications; 2001.

6 Woelbert E, Lundell-Smith K, White R, Kemmer D. Accounting for mental health research funding: developing a quantitative baseline for global investments. Lancet Psychiatry. 2021;8:250-8. Medline:33242400 doi:10.1016/S2215-0366(20)30469-7

7 Iyer AR. Authorship trends in The Lancet Global Health. Lancet Glob Health. 2018;6:e142. Medline:29389534 doi:10.1016/ S2214-109X(17)30497-7

8 Chaccour J. Authorship trends in The Lancet Global Health: only the tip of the iceberg? Lancet Glob Health. 2018;6:e497. Medline:29653622 doi:10.1016/S2214-109X(18)30110-4

9 Hedt-Gauthier BL, Jeufack HM, Neufeld NH, Alem A, Sauer S, Odhiambo J, et al. Stuck in the middle: a systematic review of authorship in collaborative health research in Africa, 2014-2016. BMJ Glob Health. 2019;4:e001853. Medline:31750000 doi:10.1136/bmjgh-2019-001853

10 Osborn TL, Wasil AR. Weisz Jr, Kleinman A, Ndetei DM. Where is the global in global mental health? A call for inclusive multicultural collaboration. Gen Psychiatr. 2020;33:e100351. Medline:33225214 doi:10.1136/gpsych-2020-100351

11 World Health Organization. WHO releases mhGAP Intervention Guide app. Available: https://www.who.int/mental_health/ mhgap/e_mhgap/en/. Accessed: 15 November 2020.

12 Godin K, Stapleton J, Kirkpatrick SI, Hanning RM, Leatherdale ST. Applying systematic review search methods to the grey literature: a case study examining guidelines for school-based breakfast programs in Canada. Syst Rev. 2015;4:138. Medline:26494010 doi:10.1186/s13643-015-0125-0

13 Collins PY, Pringle BA. Building a global mental health research workforce: perspectives from the National Institutes of Mental Health. Acad Psychiatry. 2016;40:723-6. Medline:26586615 doi:10.1007/s40596-015-0453-3

14 Mental Health Innovation Network. A global community of mental health innovators. Available: https://www.mhinnovation. net/. Accessed: 30 November 2020.

15 World Health Organization. mhGAP Forum 2017: mental health capacity building within countries. Available: https://www. who.int/mental_health/mhgap/mhGAP_forum_report_2017.pdf?ua=1. Accessed: 30 November 2020.

16 World Health Organization. World Health Organization. Available: https://www.who.int/. Accessed: 29 December 2020.

17 World Health Organization. WHO mental health newsletter. Available: https://www.who.int/mental_health/publications/mhgap_newsletters/en/. Accessed: 15 November 2020.

18 The World Bank. World Bank Country and lending groups. Available: https://datahelpdesk.worldbank.org/knowledgebase/ articles/906519. Accessed 29 December 2020.

19 World Health Organization. Chronic diseases and health promotion. Available: https://www.who.int/chp/about/regions/en/. Accessed: 16 February 2021.

20 CBM. Another step towards mental health for all. Available: https://www.cbm.org/news/news/news-2016/another-step-towardsmental-health-for-all/. Accessed: 30 November 2020. 
21 Hussain SA. Stories of success at mhGAP Kashmir: What can we learn? Available: https://www.mhinnovation.net/blog/2017/ sep/28/stories-success-mhgap-kashmir-what-can-we-learn. Accessed: 1 December 2020.

22 Syed B. Dreams do come true: How an mhGAP exchange project inspired a medical student to pursue a career in psychiatry. Available: https:/www.mhinnovation.net/blog/2017/sep/28/dreams-do-come-true-how-mhgap-exchange-project-inspired-medical-student-pursue. Accessed: 1 December 2020.

23 Tergesen C. Implicit and explicit attitudes of medical students towards mental illness: a randomized controlled pilot trial of service user videos to reduce stigma in Nepal. Available: https://dukespace.lib.duke.edu/dspace/handle/10161/16983. Accessed: 1 December 2020.

24 World Health Organization. Mental Health Gap Action Programme (mhGAP): 3rd meeting of the mhGAP Forum. Available: https://www.who.int/mental_health/mhgap/mhgap_forum_oct2011_annex_e.pdf. Accessed: 1 December 2020.

25 Chaudoir SR, Dugan AG, Barr CHI. Measuring factors affecting implementation of health inn ovations: a systematic review of structural, organizational, provider, patient, and innovation level measures. Implement Sci. 2013;8:22. Medline:23414420 doi:10.1186/1748-5908-8-22

26 Pan American Health Organization. Virtual Campus for Public Health (VCPH). Available: https://www.campusvirtualsp.org/. Accessed: 2 December 2020.

27 Musyimi C. Using mhGAP-IG on mobile phones for frontline healthcare workers to manage depression in Kenya. Available: https://www.mhinnovation.net/innovations/using-mhgap-ig-mobile-phones-frontline-healthcare-workers-manage-depression-kenya. Accessed: 1 December 2020.

28 Hamdani U. Scale-up and evaluation of family networks for children with developmental delays (FaNs for Kids). Available: https://www.mhinnovation.net/innovations/scale-and-evaluation-family-networks-children-developmental-delays-fans-kids. Accessed: 2 December 2020

29 Maulik P. SMART Mental Health. Available: https://www.mhinnovation.net/innovations/smart-mental-health. Accessed: 2 December 2020

30 MEGA. Building capacity by implementing mhGAP mobile intervention in SADC countries. Available: https://mega.turkuamk. fi/our-project/. Accessed: 2 December 2020.

31 TPO-Nepal. E-mhGAP Intervention guide in Low and middle-income countries: proof-of-concept for Impact and Acceptability (Emilia). Available: http://tponepal.org/e-mhgap-intervention-guide-in-low-and-middle-income-countries-proof-of-conceptfor-impact-and-acceptability-emilia/. Accessed: 3 December 2020.

32 UK Research and Innovation. E-MhGAP Intervention guide in low and middle income countries: proof-of-concept for Impact and Acceptability (Emilia project). Available: https://gtr.ukri.org/projects?ref=MR\%2FS001255\%2F1. Accessed: 3 December 2020.

33 Gates Cambridge Trust. GP clinics could help bridge mental health treatment gap, study finds. Available: https://www.gatescambridge.org/about/news/gp-clinics-could-help-bridge-mental-health-treatment-gap-study-finds/. Accessed: 15 December 2020.

34 Mutiso VN, Pike K, Musyimi CW, Rebello TJ, Tele A, Gitonga I, et al. Feasibility of WHO mhGAP-intervention guide in reducing experienced discrimination in people with mental disorders: a pilot study in a rural Kenyan setting. Epidemiol Psychiatr Sci. 2019;28:156-67. Medline:29862937 doi:10.1017/S2045796018000264

35 Ali S, Saeed K, Hughes P. Evaluation of a mental health training project in the Republic of the Sudan using the Mental Health Gap Action Programme curriculum. Int Psychiatry. 2012;9:43-5. Medline:31508118

36 Spagnolo J, Champagne F, Leduc N, Rivard M, Melki W, Piat M, et al. Building capacity in mental health care in low- and middle-income countries by training primary care physicians using the mhGAP: a randomized controlled trial. Health Policy Plan. 2020;35:186-98. Medline:31794027 doi:10.1093/heapol/czz138

37 Hoeft TJ, Fortney JC, Patel V, Unützer J. Task-sharing approaches to improve mental health care in rural and other low-resource settings: a systematic review. J Rural Health. 2018;34:48-62. Medline:28084667 doi:10.1111/jrh.12229

38 Raviola G, Naslund JA, Smith SL, Patel V. Innovative models in mental health delivery systems: task-sharing care with non-specialist providers to close the mental health treatment gap. Curr Psychiatry Rep. 2019;21:44. Medline:31041554 doi:10.1007/ s11920-019-1028-x

39 Uwakwe R, Otakpor A. Public mental health - using the Mental Health Gap Action Program to put all hands to the pumps. Front Public Health. 2014;2:33. Medline:24795874 doi:10.3389/fpubh.2014.00033

40 Burns JK, Tomita A. Traditional and religious healers in the pathway to care for people with mental disorders in Africa: a systematic review and meta-analysis. Soc Psychiatry Psychiatr Epidemiol. 2015;50:867-77. Medline:25515608 doi:10.1007/ s00127-014-0989-7

41 Abdulmalik J, Kola L, Fadahunsi W, Adebayo K, Taghi Yasamy M, Musa E, et al. Country contextualization of the Mental Health Gap Action Programme Intervention Guide: a case study from Nigeria. PLoS Med. 2013;10:e1001501. Medline:23976884 doi:10.1371/journal.pmed.1001501

42 Spagnolo J, Champagne F, Leduc N, Melki W, Guesmi I, Bram N, et al. Tailoring a training based on the Mental Health Gap Action Programme (mhGAP) Intervention Guide (IG) to Tunisia: process and relevant adaptations. Glob Ment Health (Camb). 2018;5:e17. Medline:29868237 doi:10.1017/gmh.2018.8

43 Bitta MA, Kariuki SM, Omar A, Nasoro L, Njeri M, Kiambu C, et al. Contextualizing and pilot testing the Mental Health Gap Action Programme Intervention Guide (mhGAP-IG) to primary healthcare workers in Kilifi, Kenya. Glob Ment Health (Camb). 2020;7:ell. Medline:32742669 doi:10.1017/gmh.2020.6

44 World Health Organization. mhGAP operations manual. Available: https://www.who.int/mental_health/mhgap/operations_ manual/en/. Accessed: 20 December 2020. 
45 Esponda GM, Harman S, Qureshi O, Sadler E, Cohen A, Kakuma R. Barriers and facilitators of mental health programmes in primary care in low-income and middle-income countries. Lancet Psychiatry. 2020;7:78-92. Medline:31474568 doi:10.1016/ S2215-0366(19)30125-7

46 Vigo D, Patten S, Pajer K, Krausz M, Taylor S, et al. Mental health of communities during the COVID-19 pandemic. Can J Psychiatry. 2020;65:681-7. Medline:32391720 doi:10.1177/0706743720926676

47 Naslund JA, Aschbrenner KA, Araya R, Marsch LA, Unützer J, Patel V, et al. Digital technology for treating and preventing mental disorders in low-income and middle-income countries: a narrative review of the literature. Lancet Psychiatry. 2017;4:486500. Medline:28433615 doi:10.1016/\$2215-0366(17)30096-2

$48 \mathrm{Fu}$ Z, Burger H, Arjadi R, Bockting CLH. Effectiveness of digital psychological interventions for mental health problems in low-income and middle-income countries: a systematic review and meta-analysis. Lancet Psychiatry. 2020;7:851-64. Medline:32866459 doi:10.1016/S2215-0366(20)30256-X

49 Hagg E, Dahinten SV, Currie LM. The emerging use of social media for health-related purposes in low and middle-income countries: A scoping review. Int J Med Inform. 2018;115:92-105. Medline:29779724 doi:10.1016/j.ijmedinf.2018.04.010

50 Spagnolo J, Champagne F, Leduc N, Melki W, Piat M, Laporta M, et al. "We find what we look for, and we look for what we know": factors interacting with a mental health training program to influence its expected outcomes in Tunisia. BMC Public Health. 2018;18:1398. Medline:30572941 doi:10.1186/s12889-018-6261-4

51 Jordans M, Breuer E. PRIME - Nepal. Available: https://www.mhinnovation.net/innovations/prime-nepal. Accessed: 15 December 2020.

52 Mental Health Innovation Network. PRIME - Uganda. Available: https://www.mhinnovation.net/innovations/prime-uganda. Accessed: 15 December 2020.

53 Mental Health Innovation Network. PRIME - Ethiopia. Available: https://www.mhinnovation.net/innovations/prime-ethiopia?qt-content_innovation=0\#qt-content_innovation. Accessed: 15 December 2020.

54 Patel V. PRIME - India. Available: https://www.mhinnovation.net/innovations/prime-india. Accessed: 15 December 2020. 